\title{
Treatment of Anorexia Nervosa in a Specialty Care Continuum
}

\author{
Teresa A. Treat, $\mathrm{PhD}^{1 *}$ \\ Elizabeth B. McCabe, PhD $^{2}$ \\ Jill A. Gaskill, CRNP ${ }^{2}$ \\ Marsha D. Marcus, PhD ${ }^{2}$
}

\begin{abstract}
Objective: Inpatient hospitalization has been a mainstay of treatment for anorexia nervosa (AN), but decreasing lengths of inpatient stay have prompted the development of alternative care-continuum models. This study characterizes the 6-month outcomes of 71 patients with AN who completed an inpatienttreatment protocol ( $\sim 5$ weeks), followed by a day-hospital program (DHP) ( 3 weeks).
\end{abstract}

Method: Data were derived from chart reviews and questionnaire data were collected during routine clinical care.

Results: On discharge from DHP, 35.2\% of patients showed excellent outcomes, whereas $23.9 \%$ exhibited poor outcomes. At 6 months after DHP discharge, $47.9 \%$ of the patients were successful in maintaining outpatient treatment status. Patients with successful 6-month outcomes were admitted to inpatient at a higher weight, had fewer previous hospitalizations, were younger, were rated by inpatient staff as more committed to treatment, endorsed less AN psychopathology during inpatient admission, and showed greater weight gain during DHP. Number of previous hospitalizations and weight gain immediately after inpatient discharge predicted $89.2 \%$ of outcome classifications 6 months after DHP discharge.

Conclusion: These findings highlight both the potential benefits of this carecontinuum model for patients who previously have not been hospitalized and the pressing need to develop alternative treatment strategies for patients with more chronic AN. (C) 2008 by Wiley Periodicals, Inc.

Keywords: anorexia nervosa; psychiatric inpatients; length of stay

(Int J Eat Disord 2008; 41:564-572)

\section{Introduction}

Anorexia nervosa (AN) is a debilitating and frequently chronic mental-health problem that affects $\sim 0.3-0.5 \%$ of the young female population. ${ }^{1-5}$ Historically, treatment of individuals with $\mathrm{AN}$ has included lengthy psychiatric hospitalizations, but the duration of inpatient stays for patients with AN has declined sharply in many regions of the country, and the ability to restore patients to normal body weight has decreased accordingly. 6 , In response to decreased duration of inpatient stays, we have developed a care continuum for patients with AN that includes inpatient hospitalization followed by a day-hospital program (DHP). In this

\footnotetext{
Accepted 2 May 2008

*Correspondence to: Teresa A. Treat, Department of Psychology, Yale University, P.O. Box 208205, New Haven, CT 06520-8205.

E-mail: teresa.treat@yale.edu

${ }^{1}$ Department of Psychology, Yale University, New Haven, Connecticut

${ }^{2}$ Department of Psychiatry, University of Pittsburgh Medical Center, Pittsburgh, Pennsylvania

Published online 20 June 2008 in Wiley InterScience (www.interscience.wiley.com). DOI: 10.1002/eat.20571

(C) 2008 Wiley Periodicals, Inc.
}

manuscript, we report on the treatment trajectory of a cohort of 71 patients with AN who were admitted to the inpatient unit and then referred for stepdown care in our DHP.

We recently reported on the development and preliminary evaluation of standardized and evidence-based clinical pathways (or treatment protocols) for the inpatient treatment of persons with AN $(n=61){ }^{8}$ Pathways were designed to resolve acute medical problems, interrupt compensatory behaviors, and initiate nutritional rehabilitation, such that patients by discharge were $\sim 85 \%$ of their ideal body mass index (BMI). Although the majority of treatment completers attained the expected treatment outcomes, patients remained ill at discharge and clearly were in need of continued intensive treatment. In our setting, patients preferably step-down to an in-house DHP, which provides close monitoring and supervised meals in the context of structured daily programming. DHPs for patients with eating disorders began appearing in the early 1980s, in response to an increasingly pressing need for cost-effective, intensive outpatient treatment alternatives to inpatient pro- 
grams. ${ }^{9,10}$ Published reports from several DHPs have indicated that patients with AN exhibit clinically significant weight gain, albeit at a much slower rate than during inpatient hospitalization, and that DHPs may provide a cost-effective freestanding or step-down approach to management of persons with AN in the current care environment. ${ }^{11-16}$

Several research teams have characterized and examined prediction of the short- and long-term outcomes of patients who received inpatient treatment for AN (see Refs. 1 and 4 for reviews of this literature). In contrast, relatively little is known about the outcomes of patients who complete briefer hospitalization stays and then step-down to brief stays in DHPs, although this combination of inpatient and intensive outpatient care is becoming an increasingly frequent treatment trajectory in the current care environment. One other report in the literature $^{14}$ presented short-term outcomes for adult female patients with AN (49 syndromal and 10 subsyndromal) who completed inpatient hospitalization and then stepped down to a DHP. Patients were discharged from inpatient after an average of 48.7 days and at an average weight of $88.7 \%$ of their ideal. Patients then completed an average of 42.6 days in a DHP that operated 7 days a week. Howard et al. ${ }^{14}$ classified $24 \%$ of their patients as treatment failures at DHP discharge, secondary to readmission to inpatient or a discharge from DHP against medical advice, indicating that a significant subset of patients responded poorly to the inpatient-DHP care continuum. Longer duration of illness and amenorrhea, as well as lower weight at inpatient admission and discharge, significantly increased the likelihood of failing treatment. Nonsignificant predictors of note included inpatient LOS and rate of weight gain, the presence of comorbid conditions, and AN subtype.

The present study aims to characterize and predict the short-term outcomes of inpatients with AN who are discharged to a DHP program in a more psychologically, medically, and nutritionally compromised state than has been reported in the research literature to date. After specifying replicable outcome criteria for patients on DHP discharge in our setting, we examine predictors of patient outcome, both upon DHP discharge and at 6 months after DHP discharge for the subset of patients who continued to receive treatment within our system of care. Potential predictors include all available clinical, historical, medical, psychological, and motivational variables. This work complements and extends the work of Howard et al. ${ }^{14}$ The length of stay in our inpatient and DHP settings was significantly shorter (33.61 and 22.03 days, respectively), and our DHP operated 5 days a week (rather than seven). Moreover, our sample included both adult and child full-syndrome patients, and we were able to examine several novel predictors of short-term outcome (e.g., psychological, motivational, and historical variables). We also examine prediction of outcomes not only at DHP discharge but also 6 months after DHP discharge.

\section{Method}

\section{Participants}

The sample consisted of 71 of the 74 unique patients who were admitted consecutively to the inpatient unit with a primary AN diagnosis and then discharged to the DHP program between January 2000 and December 2001. Two patients were excluded because they dropped out on the first day of the DHP program, and one patient was excluded because extreme diuretic misuse resulted in uninterpretable weights throughout treatment. Inpatient data from 32 of the 71 patients included in the current sample $(45.07 \%)$ were included in our previous study, ${ }^{8}$ but that study did not report any DHP data. Approval for the current project was obtained from the Biomedical Review Board of the University of Pittsburgh.

\section{Treatment}

Patients were placed on clinical pathways on the inpatient unit according to AN subtype: $63.2 \%$ were placed on the restricting-AN pathway and $36.8 \%$ were placed on the bulimic-AN pathway. The inpatient treatment program and its outcomes are described in detail in our prior work. ${ }^{8}$ The DHP offers $32 \mathrm{~h}$ of treatment from Monday to Friday, including two supervised meals and one snack each day. Close medical monitoring and nutritional counseling are mainstays of treatment, and the milieu is informed predominantly by cognitive-behavioral and dialectical-behavioral approaches. The primary treatment modality is group therapy, and pharmacotherapy is provided as indicated. Family therapy also is provided, particularly for patients under the age of 18 .

\section{Measures}

Self-Report. Patients completed three questionnaires on inpatient admission as a part of routine clinical care: (a) Eating Disorders Examination-Questionnaire (EDEQ), ${ }^{17}$ a 34 -item measure of the behavioral and psychological symptoms of eating disorders during the last 28 days; (b) Eating Disorders Inventory-2 (EDI-2), ${ }^{18}$ a 91-item scale that assesses dietary restraint, bulimic symptoms, and body dissatisfaction; and (c) Beck Depression Inven- 
tory-2 (BDI-2), ${ }^{19}$ a 21-item questionnaire designed to assess depressive symptoms. On inpatient discharge, patients again completed the EDI-2 and BDI-2; patients did not complete the EDE-Q again, because the 28-day time frame exceeded the length of stay for a number of patients. Fewer than $50 \%$ of the patients completed the EDI-2 and BDI-2 questionnaires again on DHP discharge, reflecting logistical difficulties in administering these measures in an outpatient setting in the context of routine of clinical care, so these data are not described further.

Diagnostic Information. The nurse clinical manager (JAG) of the eating-disorders unit oversaw the assignment of DSM-IV clinical diagnoses on the basis of individual interviews with patients upon admission to and discharge from the inpatient unit. Diagnoses were not reassessed on DHP discharge, given the brevity of treatment. Interviews during inpatient treatment were conducted either by the nurse manager or by a psychiatrist on the unit. Behavioral symptoms of AN, such as binge eating, purging, and ritualized eating behaviors, also were assessed during the interviews, which allowed classification of AN subtype as either purging or restricting (i.e., AN-P or AN-R) for each patient. Although interviews were conducted in a clinical rather than a research context, the assessment clinicians were well-trained and expert in the ascertainment of eating disorders diagnoses. Female patients of menarcheal age are not required to exhibit amenorrhea to receive an AN diagnosis on our unit, consistent with literature suggesting that this criterion is unnecessary and arguing that it should be dropped in DSM-V. ${ }^{20,21}$

Other Clinical Information. Historical and demographic information were obtained from patient charts at admission. Clinical staff monitored and recorded weight throughout both inpatient and day-hospital treatment. On inpatient discharge, the nurse manager rated each patient's commitment to treatment on an 11-point scale, where $0=$ "opposed to treatment throughout treatment; not committed to treatment and recovery at any point," and $10=$ "determined to make use of program throughout treatment; no ambivalence about recovery or treatment." Information on behavioral symptoms at the time of day-hospital discharge was obtained from clinician notes for a patient's final week in day hospital.

\section{Data Preparation and Analyses}

Data were complete for all variables, except for those based on the self-report questionnaires. Missing data were imputed using the Expectation-Maximization algorithm, and out-of-range estimates were replaced with the most extreme score possible for that variable. Little's test suggested that the missing data were missing completely at random, $\chi_{(211)}^{2}=238.473, p>0.05$, which justified the use of imputation methods to increase the power of analyses involving the questionnaire data.

The distributions of the majority of the variables were markedly skewed and could not be transformed to normality without discretizing the variables. Thus, all reported analyses employ nonparametric statistical methods (e.g., Mann-Whitney, Wilcoxon, McNemar, and Chi-Square tests), except as noted below. Means and standard deviations are presented in the text and tables, however, to maximize comparability with other reports. All reported $p$-values are based on two-tailed tests. All analyses were conducted with both the full sample ( $n=$ $71)$ and with the subset of patients $(n=37)$ who continued to receive treatment in our system of care after DHP discharge. The pattern of results was similar, so only the former set of analyses is reported in this paper.

Logistic regression was used to evaluate the relative and cumulative predictive power of the significant predictors of outcome at 6 months after DHP discharge. Inpatient length of stay was log-transformed to correct positive skew. The EDE Global score was skewed negatively, but its reflection could be log-transformed (and rereflected) to normality. Number of previous hospitalizations was skewed severely and could not be transformed to rough normality. Thus, this variable was discretized, by assigning values of zero (if no prior hospitalizations), one (if one prior hospitalization), or two (if two or more prior hospitalizations).

\section{Results}

\section{Demographic and Clinical Characteristics of Sample on Inpatient Admission}

The majority of participants were female (93.0\%), Caucasian (98.6\%), and single (95.7\%); average age was 18.40 years $(\mathrm{SD}=5.92)$. No prior psychiatric hospitalizations were reported by $59.2 \%$, and $28.2 \%$ reported two or more previous hospitalizations. Patients reported an average symptom duration of 3.41 years $(\mathrm{SD}=4.06)$. Restricting $\mathrm{AN}$ was diagnosed in $63.4 \%$, whereas $36.6 \%$ were diagnosed with binge-eating/purging AN. Table 1 presents additional clinical information for the full sample on inpatient admission. Average scores on the EDI2, EDE-Q, and BDI-2 at inpatient admission were similar to those for comparable samples of inpatients with eating disorders. ${ }^{14,23,24}$

\section{Pre-Post Inpatient Treatment Changes}

The average LOS on the inpatient unit was 33.61 days $(S D=17.13$, median $=28.0)$. Patients gained an average of $1.46 \mathrm{~kg}$ per week $(\mathrm{SD}=0.55)$ during inpatient hospitalization; they gained an average of 
TABLE 1. Pre-post changes during inpatient hospitalization for full sample $(n=71)$

\begin{tabular}{|c|c|c|c|c|c|}
\hline \multirow[b]{2}{*}{ Variable } & \multicolumn{2}{|c|}{ Admission } & \multicolumn{2}{|c|}{ Discharge } & \multirow[b]{2}{*}{$p$-Value } \\
\hline & Mean & SD & Mean & SD & \\
\hline $\mathrm{BMI}^{\mathrm{a}}$ & 15.19 & 1.54 & 17.56 & 1.07 & $<.05$ \\
\hline Percent-ideal BMI ${ }^{\mathrm{b}}$ & 74.08 & 7.31 & 85.62 & 4.24 & $<.05$ \\
\hline \multicolumn{6}{|l|}{ Eating disorders inventory-2 } \\
\hline Drive for thinness & 13.19 & 6.86 & 11.23 & 6.52 & $<.05$ \\
\hline Bulimia & 1.50 & 2.15 & 0.66 & 1.08 & $<.05$ \\
\hline Body dissatisfaction & 16.08 & 8.63 & 16.19 & 8.29 & n.s. \\
\hline \multicolumn{6}{|l|}{ Eating disorders examination- $\mathrm{Q}$} \\
\hline Restraint & 4.05 & 1.68 & - & & \\
\hline Weight concern & 3.76 & 1.71 & - & & \\
\hline Shape concern & 4.36 & 1.65 & - & & \\
\hline Eating concern & 3.31 & 1.52 & - & & \\
\hline Global score & 3.90 & 1.53 & - & & \\
\hline \multirow[t]{2}{*}{ Beck depression inventory-2 score } & 28.29 & 12.93 & 15.70 & 10.88 & $<.05$ \\
\hline & $N$ & $\%$ & N & $\%$ & \\
\hline \multicolumn{6}{|l|}{ Comorbid axis I psychiatric diagnoses ${ }^{\mathrm{c}}$} \\
\hline No mood or anxiety disorder ${ }^{\mathrm{d}, \mathrm{e}}$ & 32 & 45.1 & 12 & 16.9 & $<.05$ \\
\hline Only mood disorder ${ }^{\mathrm{d}}$ & 23 & 32.4 & 23 & 32.4 & n.s. \\
\hline Only anxiety disorder ${ }^{\mathrm{e}}$ & 13 & 18.3 & 19 & 26.8 & n.s. \\
\hline Both mood and anxiety disorder ${ }^{\mathrm{d}, \mathrm{e}}$ & 4 & 5.6 & 17 & 23.9 & $<.05$ \\
\hline \multicolumn{6}{|l|}{ Medication } \\
\hline Anxiolytics & 7 & 9.9 & 7 & 9.9 & n.s. \\
\hline SSRIS & 31 & 43.7 & 44 & 62.0 & $<.05$ \\
\hline Second-generation antipsychotics & 11 & 15.5 & 35 & 49.3 & $<.05$ \\
\hline \multicolumn{6}{|l|}{ Axis III problems ${ }^{\dagger}$} \\
\hline EKG changes & 49 & 69.0 & 21 & 29.6 & $<.05$ \\
\hline Electrolyte imbalances & 8 & 11.3 & 0 & 0.0 & $<.05$ \\
\hline Kidney function problems & 22 & 31.0 & 2 & 2.8 & $<.05$ \\
\hline Liver function problems & 17 & 23.9 & 5 & 7.0 & $<.05$ \\
\hline Phosphorus/magnesium problems & 11 & 15.5 & 4 & 5.6 & $<.05$ \\
\hline Neutropenia & 16 & 22.5 & 5 & 7.0 & $<.05$ \\
\hline
\end{tabular}

Notes: SSRI, selective serotonin reuptake inhibitors; BMI, body mass index.

${ }^{a}$ Weight $(\mathrm{kg}) /$ height $^{2}(\mathrm{~m})$.

${ }^{\mathrm{b}}$ Absolute body mass index divided by the median body mass index for a given age and sex, as specified on Centers for Disease Control ${ }^{22}$ growth charts.

${ }^{c}$ The nurse clinical manager (J.A.G.) of the eating-disorders unit oversaw the assignment of DSM-IV clinical diagnoses on the basis of individual interviews with patients at admission to and discharge from the inpatient unit.

${ }^{\mathrm{d}}$ Major depressive disorder with or without psychotic features, depressive disorder NOS, dysthymia.

e Obsessive-compulsive disorder, generalized anxiety disorder, panic disorder, post-traumatic stress disorder, social phobia, specific phobia, anxiety disorder NOS.

${ }^{f}$ The specific medical problems included in the first five categories of Axis III problems are listed in Table 5 in Treat et al. (2005). ${ }^{8}$

$1.38 \mathrm{~kg}$ during the first week $(\mathrm{SD}=1.10)$. Table 1 presents changes in BMI, percent-ideal BMI, questionnaire data, comorbid diagnoses, medication usage, and medical problems. Note that the increase in the number of Axis I diagnoses during inpatient treatment reflects unit policy to defer additional diagnoses until after patient stabilization and the initiation of renutrition, unless pre-existing information about the patient is available. The average treatment commitment rating at inpatient discharge was $5.11(\mathrm{SD}=2.13)$.

\section{Pre-Post DHP Treatment Changes}

The average LOS in DHP was 22.03 days (SD = 13.38 , median $=19$ ). Patients gained an average of $0.24 \mathrm{~kg}$ per week $(\mathrm{SD}=0.77$, median $=0.34)$ during their DHP stay, and they gained an average of 0.16 $\mathrm{kg}(\mathrm{SD}=0.91)$ during the first 5 days after inpatient discharge. $\mathrm{BMI}$ increased from $17.62(\mathrm{SD}=1.08)$ to
17.90 (SD = 1.07), $Z=-3.16, p<.01$. Percent-ideal BMI increased from $85.91(\mathrm{SD}=4.43)$ to 87.33 (SD $=$ 5.77), $Z=-3.22, p<.01$. This increase in average BMI reflects a mean weekly weight gain of $0.24 \mathrm{~kg}$ (median $=0.34 \mathrm{~kg}$ ) during the 3 -week period, which falls within the recommended range of weight gain per week for patients being treated in ambulatory settings $\left(0.5-1.0\right.$ pounds per week $\left.^{25}\right)$.

\section{Prediction of DHP Outcome from Inpatient Information}

Outcome classification criteria at DHP discharge were based on weight and the presence of compensatory behaviors, two commonly utilized criteria included in the definition of short-term recovery. ${ }^{26}$ To be classified as exhibiting either an excellent or good outcome, patients could not be losing more than one-third a pound per week, as our clinical experience has suggested that even minimal weight 
loss after inpatient treatment is a harbinger of potential deterioration in clinical status. Psychological symptoms of AN are also key indicators of recovery; however, we obtained this information on too few patients at the time of DHP discharge to incorporate psychological symptoms into our classification scheme. Table 2 summarizes our shortterm outcome criteria.

Patient DHP outcomes were classified as $35.2 \%$ excellent, $26.8 \%$ good, $14.1 \%$ below average, and $23.9 \%$ poor. All variables obtained during the inpatient admission were examined as potential predictors of DHP outcome classification; good and

TABLE 2. Outcome classification criteria on discharge from day hospital program

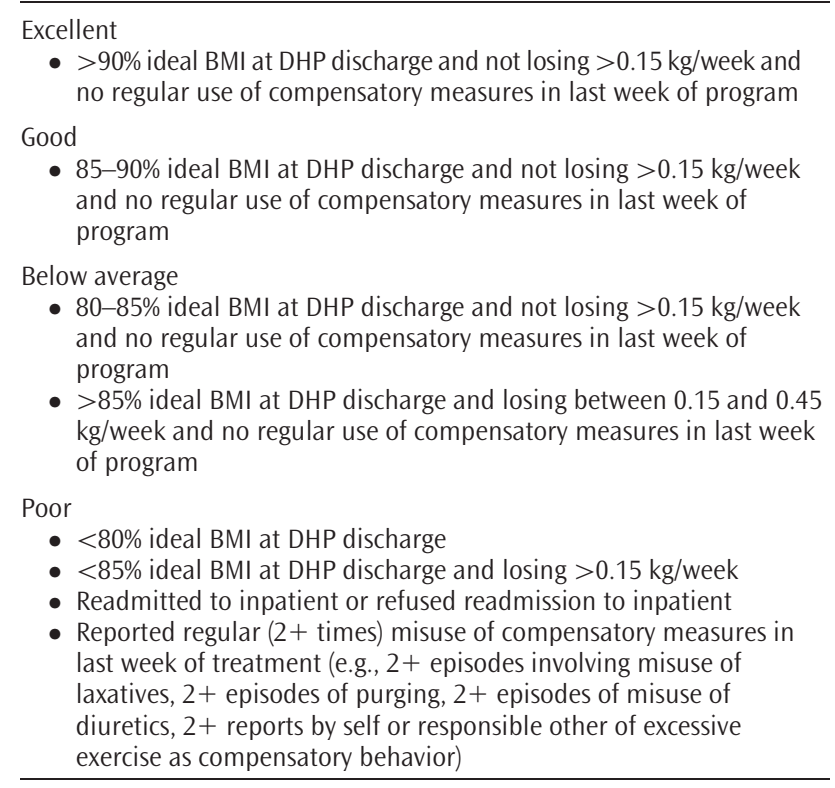

Note: BMI, body mass index; DHP, day hospital program. below-average categories were collapsed into an intermediate category for these analyses. Table $\mathbf{3}$ presents descriptive information for patients in the three outcome categories for all significant and trendlevel predictors. Nonsignificant predictors of note included the presence of comorbid mood or anxiety disorders, medication regimen or medical problems, inpatient LOS, AN subtype, and clinician ratings of treatment commitment on inpatient discharge.

\section{Prediction of 6-Month Outcome Classifications}

Treatment within our hospital system after DHP discharge, $52.1 \%$ of the patients $(n=37)$ continued treatment within our hospital system, whereas the remaining patients received care elsewhere after DHP discharge. Patient outcomes were classified as unsatisfactory ( $n=19,51.35 \%) 6$ months after DHP discharge if patients had been referred by their individual clinician in the eating-disorders program to a higher level of care (i.e., an intensive outpatient, day hospital, residential, or inpatient program). Such referrals occurred because of significant weight loss, regular use of compensatory behaviors, medical instability, or significant suicidal intent. Thus, this criterion ensured that all patients with clearly unsatisfactory outcomes were classified appropriately. Otherwise, patient outcomes were classified as satisfactory $(n=18$, 48.65\%). Table 4 presents all significant and trendlevel differences between these two groups on all variables that were available from either inpatient or DHP treatment.

A forward-stepwise logistic-regression method using the Wald score as an entry criterion was used to select variables for inclusion in the model from the pool of six potential predictors. To avoid multi-

TABLE 3. Prediction of outcome classifications at day-hospital program discharge for full sample $(n=71)$

\begin{tabular}{|c|c|c|c|}
\hline Variable & Excellent $(n=25)$ & Intermediate $(n=29)$ & $\operatorname{Poor}(n=17)$ \\
\hline $\operatorname{Age}^{\mathrm{a}}$ & $15.40(2.69)^{\mathrm{d}, \mathrm{e}}$ & $18.92(6.47)^{\mathrm{d}}$ & $21.93(6.42)^{\mathrm{e}}$ \\
\hline Previous ED hospitalizations ${ }^{a}$ & $0.60(1.35)^{\mathrm{d}}$ & $0.93(1.71)^{\mathrm{e}}$ & $2.59(3.14)^{d, e}$ \\
\hline Duration of eating disorder (years) ${ }^{\mathrm{a}}$ & $1.47(1.32)^{\mathrm{d}, \mathrm{e}}$ & $3.13(3.11)^{d}$ & $6.74(5.90)^{\mathrm{e}}$ \\
\hline \multicolumn{4}{|l|}{ Percent-ideal BMI ${ }^{\mathrm{e}}$} \\
\hline Inpatient admission ${ }^{b}$ & $76.86(4.87)^{\mathrm{d}}$ & $73.23(7.49)$ & $71.42(8.89)^{\mathrm{d}}$ \\
\hline Inpatient discharge ${ }^{a}$ & $88.26(3.09)^{\mathrm{d}, \mathrm{e}}$ & $84.48(3.06)^{\mathrm{e}}$ & $83.66(5.53)^{d}$ \\
\hline \multicolumn{4}{|l|}{ Eating disorders inventory } \\
\hline Drive for thinness at inpatient admission ${ }^{a}$ & $12.49(7.49)$ & $11.85(6.41)^{\mathrm{d}}$ & $16.51(5.85)^{d}$ \\
\hline Body dissatisfaction at inpatient admission ${ }^{a}$ & $15.58(10.12)$ & $13.98(7.45)^{d}$ & $20.41(6.85)^{d}$ \\
\hline Drive for thinness at inpatient discharge ${ }^{a}$ & $9.62(7.66)^{d}$ & $10.37(5.76)^{\mathrm{e}}$ & $15.09(4.59)^{\mathrm{d}, \mathrm{e}}$ \\
\hline Body dissatisfaction at inpatient discharge ${ }^{a}$ & $13.50(9.60)^{d}$ & $15.39(7.54)^{\mathrm{e}}$ & $21.50(4.52)^{\mathrm{d}, \mathrm{e}}$ \\
\hline \multicolumn{4}{|l|}{ Beck depression inventory-2 } \\
\hline Inpatient discharge $\mathrm{a}^{\mathrm{a}}$ & $14.51(13.79)^{\mathrm{d}}$ & $13.78(8.78)^{\mathrm{e}}$ & $20.73(7.90)^{\mathrm{d}, \mathrm{e}}$ \\
\hline \multicolumn{4}{|l|}{ Eating disorders examination-Q } \\
\hline Global score ${ }^{b}$ & $3.40(1.95)$ & $3.86(1.25)^{d}$ & $4.70(0.84)^{\mathrm{d}}$ \\
\hline
\end{tabular}

Notes: Group pairs with the same subscript differ significantly, using Mann-Whitney test to compare group medians; BMI, body mass index.

a Significant group difference $(p<.05)$, using Kruskal-Wallis test to compare group medians.

${ }^{\mathrm{b}}$ Nonsignificant tendency toward group difference $(p<.10)$, using Kruskal-Wallis test to compare group medians.

${ }^{c}$ Absolute body mass index divided by the median body mass index for a given age and sex, as specified on Centers for Disease Control ${ }^{22}$ growth charts. 
TABLE 4. Predictors of 6-month outcome after discharge from day-hospital program for subsample remaining in treatment system $(n=37)$

\begin{tabular}{|c|c|c|c|c|}
\hline \multirow[b]{2}{*}{ Variable } & \multicolumn{2}{|c|}{ Satisfactory $(n=18)$} & \multicolumn{2}{|c|}{ Unsatisfactory $(n=19)$} \\
\hline & Mean & SD & Mean & SD \\
\hline Inpatient length of stay (days) ${ }^{a}$ & 23.06 & 8.77 & 33.00 & 14.03 \\
\hline Previous psychiatric hospitalizations for primary eating disorder ${ }^{a}$ & 0.33 & 0.77 & 2.26 & 3.19 \\
\hline Average weight gain per week in DHP $(\mathrm{kg})^{\mathrm{a}}$ & 0.51 & 0.63 & -0.21 & 1.00 \\
\hline Weight change in first 5 days after inpatient discharge $(\mathrm{kg})^{\mathrm{a}}$ & 0.55 & 0.74 & -0.31 & 0.88 \\
\hline Treatment commitment at inpatient discharge ${ }^{\mathrm{a}}$ & 5.95 & 2.14 & 4.18 & 2.60 \\
\hline \%-ideal $\mathrm{BMI}^{\mathrm{C}}$ at inpatient admission ${ }^{\mathrm{a}}$ & 78.52 & 3.83 & 73.43 & 6.81 \\
\hline $\operatorname{Age}^{\mathrm{b}}$ & 16.29 & 3.03 & 18.90 & 5.02 \\
\hline \multicolumn{5}{|l|}{ Eating disorders inventory } \\
\hline Body dissatisfaction at inpatient discharge $\mathrm{e}^{\mathrm{b}}$ & 14.22 & 9.19 & 18.99 & 7.55 \\
\hline \multicolumn{5}{|l|}{ Eating disorders examination-Q } \\
\hline Global score ${ }^{b}$ & 3.27 & 1.86 & 4.43 & 1.31 \\
\hline
\end{tabular}

collinearity problems, inpatient LOS and the EDE global score were not included in the logistic regression analysis. The Hosmer and Lemeshow goodness-of-fit test indicated that the model provided an adequate fit to the data, $\chi_{(7)}^{2}=12.407, p>$ .05 ; the Nagelkerke $R^{2}$ indicated that the model accounted for $50.5 \%$ of the variability in outcome status. Variables were retained in the final model if they accounted for significant variability in outcome status above and beyond that accounted for by previously retained variables. Number of prior hospitalizations and change in weight during the first 5 days after inpatient discharge were retained in the final model, Wald statistics $=6.041$ and $6.597, p<.05$, respectively. These two variables correctly predicted the outcome status of 17 of the 18 patients $(94.4 \%)$ with satisfactory outcomes and 16 of the 19 patients $(84.2 \%)$ with unsatisfactory outcomes. Cutoff scores that optimized the relative risk of an unsatisfactory outcome were computed for both significant contributors to the multivariate model. These computations indicated that patients who previously had been hospitalized two or more times were 6.3 times more likely to show an unsatisfactory outcome and those patients who gained less than a pound in the first 5 days after inpatient discharge were 4.2 times more likely to show an unsatisfactory outcome.

\section{Conclusion}

The current investigation aimed to provide a comprehensive depiction of the short-term outcomes of a sizeable sample of patients with AN $(n=71)$ who completed a care continuum designed in response to decreasing lengths of inpatient stay. Patients completed a standardized and evidence-informed inpatient-treatment protocol for $\sim 5$ weeks, followed by a DHP for $\sim 3$ weeks. Our findings document that most patients can meet modest inpatient goals during a relatively brief inpatient stay, but they remain acutely ill upon discharge and are in need of continued intensive care. Nevertheless, the integrated combination of inpatient hospitalization and DHP is sufficient to provide excellent shortterm outcomes for a sizeable subsample of patients who have not been hospitalized previously and who continue to gain weight after inpatient discharge. Unfortunately, however, the care continuum is less effective for patients with more chronic AN who do not gain weight after inpatient discharge. Finally, outcome classification at 6 months after completion of inpatient and DHP can be predicted extremely well by the combination of number of prior hospitalizations and amount of weight gained in the first few days after inpatient discharge.

\section{Inpatient Hospitalization Outcomes}

The findings of this investigation replicate those we reported in an earlier study. ${ }^{8}$ Patients were $\sim 85 \%$ of their ideal BMI by the end of inpatient hospitalization, having gained an average of almost $1.5 \mathrm{~kg}$ per week. Acute medical problems had resolved, the use of compensatory measures had ceased, and several self-reported psychological aspects of the illness had improved, including the urges to restrict and engage in bulimic behaviors, as well as depressive symptoms. Nutritional rehabilitation was far from complete on inpatient discharge, and significant medical problems persisted. Moreover, patients' self-reported body dissatisfaction remained unchanged, and significant de- 
creases in other aspects of AN psychopathology were smaller in magnitude than the reductions observed in investigations evaluating longer interventions. ${ }^{23,24,27-30}$ Clinician ratings also indicated that the average patient showed marked ambivalence about treatment and recovery at inpatient discharge. Thus, it is critical to continue intensive treatment and to examine outcome trajectories after inpatient discharge.

\section{Day Hospital Program Outcomes}

Although patients were still acutely ill on admission to DHP, $35.2 \%$ of the patients at DHP discharge were classified as showing an excellent outcome. Predictor analyses indicated that these patients were more likely to be younger, with fewer previous hospitalizations and a shorter duration of illness; showed a higher weight at inpatient discharge; and reported less psychological distress at inpatient discharge. Thus, a subset of patients does very well with inpatient care, followed by an average of three additional weeks in DHP.

This encouraging finding does not obviate the fact that $23.9 \%$ of the patients were classified as exhibiting an unsatisfactory outcome at DHP discharge. Predictor analyses indicated that these patients tended to be older, had more chronic AN, weighed less at inpatient discharge, and reported greater psychological distress at inpatient admission and discharge. Thus, this particular care-continuum model clearly is inadequate to avert treatment failure for these patients, consistent with numerous reports that have documented high rates of treatment failure for this subpopulation, as well as protracted recovery trajectories. Additional work is needed to consider further how best to adapt treatment planning for patients with chronic AN, which might or might not involve pressing for longer length of inpatient stay.

A comparison of the findings observed in the present study and in the study conducted by Howard et al. ${ }^{14}$ reveals two notable points of convergence, even though our DHP operated only 5 days a week (rather than seven) and the average length of stay in our inpatient and DHP was considerably briefer. First, Howard et al. ${ }^{14}$ also classified $24 \%$ of their patients as treatment failures after completing inpatient treatment and then DHP, reinforcing our observation that a significant subset of patients respond poorly to the inpatient-DHP care continuum. Second, the pattern of predictors of outcome for the two care-continuum models was similar across the two studies. Overlapping predictors included duration of illness and weight at inpatient admission (at a trend level in our study) and discharge. Nonsignificant predictors that emerged across the two samples included inpatient LOS and rate of weight gain, comorbid conditions, and AN subtype. Overall, therefore, the present study replicated and extended the previous outcome data and pattern of predictors.

Of the 37 patients (52.1\% of our total sample) who continued to receive treatment in our system after DHP discharge, $51.4 \%$ were referred back to a higher level of care within 6 months. The high percentage of treatment failures is of concern, but perhaps indicative of AN treatment outcomes in our current care climate, as well as consistent with reports documenting a protracted course of recovery for many patients with AN. ${ }^{31}$ Univariate predictor analyses documented that numerous individual factors were associated with favorable outcomes including younger age, fewer previous hospitalizations, higher inpatient admission weights, higher staff ratings of treatment commitment at inpatient discharge, less severe eating-related psychopathology on inpatient admission and discharge (at trend levels), continued weight gain immediately after inpatient discharge and throughout the DHP. Importantly, multivariate analyses revealed that the best predictors of treatment outcome 6 months after DHP discharge were number of prior psychiatric hospitalizations for an eating disorder and weight change in the first 5 days after inpatient discharge. Multivariate prediction of outcome on the basis of only these two variables was excellent and afforded accurate prediction of outcome status for almost $90 \%$ of the patients.

The current investigation has several significant strengths, including the study of a sizable sample of full-syndrome patients with AN who received best-practice treatment in inpatient and then dayhospital settings. In the current care environment of increasingly abbreviated inpatient stays, this treatment trajectory is becoming more common, but we are aware of only one other report ${ }^{14}$ presenting outcome information for patients who complete this treatment sequence. Nevertheless, significant limitations also are evident in the present work. Most notably, it was not possible to use structured diagnostic interview procedures to ascertain clinical diagnoses. Additionally, we were unable to obtain information on 6-month outcomes for patients who completed inpatient and day-hospital treatment but then received continuing treatment in other facilities. In spite of these weaknesses, however, the current work provides a novel and informative addition to the AN treatment-outcome literature. 


\section{Conclusions and Implications}

The findings in the current study reinforce other findings in the literature in suggesting that the weights at which patients are admitted to and discharged from inpatient be increased, as weight at both time points is a strong correlate of short-term outcome. ${ }^{14,32}$ Young patients who previously have not been hospitalized were more likely to show positive short-term outcomes. Thus, these data suggest the importance of treating these patients aggressively. Conversely, efforts to develop interventions for older patients and those who are refractory to front-line interventions also are of paramount importance.

Eating-related psychological aspects of AN, such as dietary restraint and body dissatisfaction, merit closer consideration in future work and when considering inpatient LOS. Data from our center reported previously ${ }^{8}$ and in the present study revealed nonsignificant or negligible drops in these indices during hospitalization. These are anomalous findings in the AN inpatient treatment-outcome literature $\mathrm{e}^{23,24,27-30}$ that may be attributable to the shorter duration of inpatient stays or to the lower weights at which inpatients were discharged. These findings, in concert with the relationships between AN psychopathology and short-term outcome that emerged in the present study, are consistent with observations that patients diagnosed with AN may be particularly vulnerable during the period when there has been some physical recovery, but before substantial psychological improvement has occurred. ${ }^{33}$ Consequently, as we may be discharging patients to DHP during this period of heightened vulnerability, work is needed to determine the degree of improvement in AN psychopathology that is consistent with the likelihood of a continuing trajectory of recovery after discharge to a less intensive level of care.

Both average weight gain per week during DHP and weight change in the first 5 days after inpatient discharge emerged as strong predictors of outcome status 6 months after DHP discharge, with the latter emerging as one of the two best predictors of outcome status in multivariate analyses. In contrast, neither comparable index of weight gain during inpatient hospitalization predicted outcome. This pattern of results suggests that the first few days after inpatient discharge may be a high-risk period, presumably secondary to the sharp reduction in structure, support, and supervision provided by the inpatient milieu. Although weight change during this period may be little more than a proxy for other variables, it also may be an early and partly independent signal of later treatment failure. Unlike other predictors of short-term outcome, such as duration of illness, weight on inpatient admission and discharge, and psychological variables, weight change during the first few days after inpatient discharge is potentially amenable to intervention. A comprehensive examination of the problematic circumstances that patients, families, and staff encounter during this time period, followed by structured provision of this information and necessary training in the implementation of effective solutions to commonly encountered difficulties, might help to avert treatment failure among some patients.

Overall, the present study has provided preliminary support for a care-continuum model of treatment for patients diagnosed with $\mathrm{AN}$ in an era in which inpatient length of stay has declined sharply, particularly for younger patients who have not been hospitalized previously, who are admitted to and discharged from inpatient at higher weights, who endorse less AN psychopathology on inpatient admission and discharge, who exhibit a greater commitment to treatment and recovery, and who continue to gain weight after inpatient discharge. Future research should explore alternative treatment options for patients with chronic $\mathrm{AN}$ and continue to develop and evaluate methods to address the psychological aspects of AN, to enhance motivation to change, and to improve rates of weight gain immediately after inpatient discharge. Characterizing and predicting the longterm outcomes associated with this care-continuum model also should be a priority, as we need to determine whether a subset of patients continues to succeed in the long term and to evaluate whether the pattern of predictors changes as a function of time since DHP discharge.

Preparation of this manuscript was supported in part by a Pilot/Feasibility award from the University of Pittsburgh Obesity/Nutrition Research Center (Pittsburgh PA, USA) to the first and last authors. We thank Anna Bardone-Cone for her helpful contributions to this project and V. Robin Weersing for discussions related to this manuscript. Requests for a copy of the clinical pathways should be addressed to Marsha D. Marcus, Western Psychiatric Institute and Clinic, 3811 O'Hara Street, Pittsburgh PA 15213, or via electronic mail to marcusmd@upmc.edu.

\section{Earn CE credit for this article!}

Visit: http://www.ce-credit.com for additional information. There may be a delay in the posting of the article, so continue to check back and look for the section on Eating Disorders. Additional information about the program is available at www.aedweb.org 


\section{References}

1. Pike KM. Long-term course of anorexia nervosa: Response, remission, and recovery. Clin Psychol Rev 1998;18:447-475.

2. Löwe B, Zipfel S, Buchholz C, Dupont Y, Reas DL, Herzog W. Long-term outcome of anorexia nervosa in a prospective 21year follow-up study. Psychol Med 2001;31:881-890.

3. Wilson GT, Pike KM. Eating disorders. In: Barlow DH, editor. Clinical Handbook of Psychological Disorders: A Step-by-Step Treatment Manual, 3rd ed. New York, NY: The Guilford Press, 2001, pp. 332-375.

4. Steinhausen $\mathrm{H}$. The outcome of anorexia nervosa in the $20^{\text {th }}$ century. Am J Psychiatry 2002;159:1284-1293.

5. Hoek HW. Incidence, prevalence and mortality of anorexia nervosa and other eating disorders. Curr Opin Psychiatry 2006;19:389-394.

6. Kaye WH, Enright AB, Lesser S. Characteristics of eating disorders programs and common problems with third-party providers. Int J Eat Disord 1998;7:573-579.

7. Wiseman CV, Sunday SR, Klapper F, Harris WA, Halmi KA. Changing patterns of hospitalization in eating disorder patients. Int J Eat Disord 2001;30:69-74.

8. Treat TA, Gaskill JA, McCabe EB, Ghinassi FA, Luczak AD, Marcus MD. Short-term outcome of psychiatric inpatients with anorexia nervosa in the current care environment. Int J Eat Disord 2005;38:123-133.

9. Meads C, Gold L, Burls A. How effective is outpatient care compared to inpatient care for the treatment of anorexia nervosa? A systematic review. Eur Eat Disord Rev 2001;9:229-241.

10. Zipfel S, Reas DL, Thornton C, Olmsted MP, Williamson DA, Gerlinghoff $M$, Herzog W, Beumont PJ. Day hospitalization programs for eating disorders: A systematic review of the literature. Int J Eat Disord 2002;31:105-117.

11. Kaplan AS, Olmsted MP. Partial hospitalization. In: Garner DM, Garfinkel PE, editors. Handbook of Treatment for Eating Disorders, 2nd ed. New York, NY: The Guilford Press, 1997, pp. 354-360.

12. Gerlinghoff $M$, Backmund $H$, Franzen $U$. Evaluation of a day treatment programme for eating disorders. Eur Eat Disord Rev 1998;6:96-106.

13. Williamson DA, Duchmann EG, Barker SE, Bruno RM. Anorexia nervosa. In: Van Hasslet VB, Hersen M, editors. Handbook of Psychological Treatment Protocols for Children and Adolescents. Hillsdale, NJ: Lawrence Erlbaum Associates, 1998, pp. 413-434.

14. Howard WT, Evans KK, Quintero-Howard CV, Bowers WA, Anderson A. Predictors of success or failure of transition to day hospital treatment for inpatients with anorexia nervosa. Am J Psychiatry 1999;156:1697-1702

15. Olmsted MP, McFarlane T, Molleken L, Kaplan AS. Day hospital treatment of eating disorders. In: Gabbard GO, editor. Treatments of Psychiatric Disorders. Washington, DC: American Psychiatric Publishing, 2001, pp. 2127-2137.
16. Williamson DA, Thaw JM, Varnado-Sullivan PJ. Cost-effectiveness analysis of a hospital-based cognitive-behavioral treatment program for eating disorders. Behav Ther 2001;32:459-477.

17. Fairburn CG, Beglin SJ. Assessment of eating disorders: Interview or self-report questionnaire? Int J Eat Disord 1994;16:363370 .

18. Garner DM. Eating Disorder Inventory-2: Professional Manual. Odessa, FL: Psychological Assessment Resources, 1991.

19. Beck AT, Steer RA, Brown GK. Manual for Beck Depression Inventory-II. San Antonio, TX: Psychological Corp., 1996.

20. Cachelin FM, Maher BA. Is amenorrhea a critical criterion for anorexia nervosa? J Psychosom Res 1998;44:435-440.

21. Mitchell JE, Cook-Myers T, Wonderlich SA. Diagnostic criteria for anorexia nervosa: Looking ahead to DSM-V. Int I Eat Disord 2005;37S:S95-S97.

22. Centers for Disease Control. 2000 CDC Growth Charts: United States. Available at: http://www.cdc.gov/growthcharts. Accessed Dec 31, 2000

23. Probst M, Vandereycken W, Van Coppenolle H, Pieters G. Body experience in eating disorders before and after treatment: A follow-up study. Eur Psychiatry 1999;14:333-340.

24. Pike KM. How do we keep patients well: Issues of relapse prevention. Paper presented at the International Conference on Eating Disorders 2000. New York, NY.

25. American Psychiatric Association. Treatment of patients with eating disorders, third edition. Am J Psychiatry 2006;163(7 Suppl):4-54.

26. Mclntosh VV, Jordan J, Carter FA, Luty SE, McKenzie JM, Bulik CM, Frampton CMA, Joyce PR. Three psychotherapies for anorexia nervosa: A randomized, controlled trial. Am J Psychiatry 2005;162:741-747.

27. Channon S, DeSilva WP. Psychological correlates of weight gain in patients with anorexia nervosa. J Psychiatr Res 1985;19:267271.

28. Steinhausen H. Evaluation of inpatient treatment of adolescent anorexic patients. J Psychiatr Res 1985;19:371-375.

29. Grave RD, Bartocci C, Todisco P, Pantano M, Bosello O. Inpatient treatment for anorexia nervosa: A lenient approach. Eur Eat Disord Rev 1993;1:166-176.

30. Bowers WA, Ansher LS. Cognitions in anorexia nervosa: Changes at discharge from a cognitive therapy milieu inpatient treatment program. J Cogn Psychother Int Q 2000;14:393-401.

31. Strober M, Freeman R, Morrell W. The long-term course of severe anorexia nervosa in adolescents: Survival analysis of recovery, relapse, and outcome predictors over 10-15 years in a prospective study. Int J Eat Disord 1997;22:339-360.

32. Baran SA, Weltzin TE, Kaye WH. Low discharge weight and outcome in anorexia nervosa. Am J Psychiatry 1995;152:10701072

33. Fennig S, Fennig S, Roe D. Physical recovery in anorexia nervosa: Is this the sole purpose of a child and adolescent medicalpsychiatric unit? Gen Hosp Psychiatry 2002;24:87-92. 